OPEN ACCESS

University of Dundee

\title{
A Prototype Therapeutic Capsule Endoscope for Ultrasound-mediated Targeted Drug Delivery
}

Stewart, Fraser; Verbeni, Antonella ; Qiu, Yongqiang ; Cox, Benjamin; Vorstius, Jan; Newton, Ian

Published in:

Journal of Medical Robotics Research

DOI:

$10.1142 / \mathrm{S} 2424905 \mathrm{X} 18400019$

Publication date:

2018

Document Version

Peer reviewed version

Link to publication in Discovery Research Portal

Citation for published version (APA):

Stewart, F., Verbeni, A., Qiu, Y., Cox, B., Vorstius, J., Newton, I., Huang, Z., Menciassi, A., Nathke, I., \& Cochran, S. (2018). A Prototype Therapeutic Capsule Endoscope for Ultrasound-mediated Targeted Drug Delivery. Journal of Medical Robotics Research, 3(2), [1840001]. https://doi.org/10.1142/S2424905X18400019

\section{General rights}

Copyright and moral rights for the publications made accessible in Discovery Research Portal are retained by the authors and/or other copyright owners and it is a condition of accessing publications that users recognise and abide by the legal requirements associated with these rights.

- Users may download and print one copy of any publication from Discovery Research Portal for the purpose of private study or research.

- You may not further distribute the material or use it for any profit-making activity or commercial gain.

- You may freely distribute the URL identifying the publication in the public portal.

Take down policy

If you believe that this document breaches copyright please contact us providing details, and we will remove access to the work immediately and investigate your claim. 


\title{
A Prototype Therapeutic Capsule Endoscope for Ultrasound-mediated Targeted Drug Delivery
}

\author{
Fraser Stewart ${ }^{\mathrm{a},}$, Antonella Verbeni ${ }^{\mathrm{b}}$, Yongqiang Qiu ${ }^{\mathrm{c}}$, Ben F Cox ${ }^{\mathrm{d}}$, Jan Vorstius ${ }^{\mathrm{e}}$, Ian P Newton ${ }^{\mathrm{a}}$, Zhihong \\ Huang $^{\mathrm{e}}$, Arianna Menciassi ${ }^{\mathrm{b}}$, Inke Näthke ${ }^{\mathrm{a}}$, Sandy Cochran ${ }^{\mathrm{c},}{ }^{+}$ \\ ${ }^{a}$ School of Life Sciences, University of Dundee, Dundee, DD1 5EH, Scotland \\ ${ }^{b}$ The BioRobotics Institute, Scuola Superiore Sant'Anna, Pisa, Italy \\ ${ }^{c}$ School of Engineering, University of Glasgow, Glasgow, G12 8QQ, Scotland \\ ${ }^{d}$ School of Medicine, University of Dundee, Dundee, DD1 9SY, Scotland \\ ${ }^{e}$ School of Science and Engineering, University of Dundee, Dundee, DDI 4HN, Scotland
}

\begin{abstract}
The prevalence of gastrointestinal (GI) diseases such as Crohn's disease, which is chronic and incurable, are increasing worldwide. Treatment often involves potent drugs with unwanted side effects. The technological - pharmacological combination of capsule endoscopy with ultrasoundmediated targeted drug delivery (UmTDD) described in this paper carries new potential for treatment of these diseases throughout the GI tract. We describe a proof-of-concept UmTDD capsule and present preliminary results to demonstrate its promise as an autonomous tool to treat GI diseases.
\end{abstract}

Keywords: Capsule Endoscopy, Therapy, Focused Ultrasound, Drug Delivery, UmTDD, Ultrasound Capsule Endoscopy (USCE)

\section{Introduction}

Diseases of the gastrointestinal (GI) tract, such as inflammatory bowel disease, present a major health problem in the developed world, with more than one million people in the USA and 2.5 million in Europe affected ${ }^{1}$. A prominent example is Crohn's disease, a condition affecting 700,000 people in the USA and 1.5 million people in Europe at present. Crohn's disease is chronic and incurable, with treatment aiming to suppress symptoms and achieve remission. The drugs in use range from steroids to more potent biological therapies delivered either orally or systemically. In each case they are associated with undesirable side effects and damage to healthy tissue.

To make treatment more effective, earlier diagnosis is required to minimize complications. Crohn's disease is most prevalent in the small intestine and diagnosis by endoscopy is difficult since this technique does not readily reach the small intestine. An alternative is video capsule endoscopy (VCE); this involves ingesting a small, autonomous capsule containing a light source, camera and wireless communication capability. VCE has many advantages over traditional optical endoscopy: it can visualize the entire GI tract; it is easier to tolerate and less invasive for patients; it does not require sedation and it only requires a skilled user to analyze the images rather than a skilled, highly experienced operator ${ }^{2}$. These advantages have motivated its use in more than 1.5 million procedures to date worldwide ${ }^{3}$. However, VCE also has some disadvantages: localization is poor; capsule positioning is not possible as the capsule currently relies on physiological motion to move through the GI tract; and retention is possible, particularly in Crohn's disease and other forms of inflammatory bowel disease that can cause strictures.

Including modalities in addition to video imaging has been proposed to make capsule endoscopy more useful. One example is "Sonopill"4 (www.glasgow.ac.uk/sonopill), a research programme that aims to combine video imaging with other diagnostic modalities such as microultrasound and fluorescence imaging to allow diagnosis of Crohn's disease and other diseases. A distinct advantage provided by these modalities is the ability to image the entire depth of the wall of the GI tract, beyond the optically accessible mucosal surface.

There are also examples of therapeutic capsule endoscopes (TCE) that have the potential to deliver therapy, by acting as drug delivery devices. Ablation is another possibility that can be included in TCE but associated high power demands make it difficult to deliver it in capsule format and this approach also carries a risk of perforation and peritonitis. Examples of drug delivery capsule devices are the Enterion ${ }^{5}$ and the Intelisite ${ }^{6}$ capsules. Both contain a reservoir with a capacity of approximately $1 \mathrm{ml}$ that can be emptied by a remote trigger. However, neither can be localized or positioned accurately nor

Email Addresses: $\quad *$ fwstewart@dundee.ac.uk

† Sandy.Cochran@glasgow.ac.uk 
can they deliver drugs through the mucosa, limiting their efficacy. There have also been attempts to combine drug delivery with positioning and localization functions ${ }^{7,8}$, however these capsules lack the ability to enhance uptake into and through the mucosa. A capsule containing a microneedle to deliver drugs into the mucosa has also been developed ${ }^{9}$. However, it also cannot be localized or positioned precisely and the microneedle could potentially perforate the mucosa.

One solution to these challenges in TCE is to use an ultrasound (US)-mediated targeted drug delivery (UmTDD) system. UmTDD could deliver therapeutics at a target location, using focused US to release therapeutics from carriers and/or to increase drug uptake through the mechanical or thermal effects of US. One of the useful bioeffects of focused US is cavitation, the stimulation of small gas bubbles exposed to an ultrasound pressure field. There are two types of cavitation: stable and inertial. In stable cavitation bubbles oscillate but do not rupture and in inertial cavitation oscillations reach a level that causes bubbles to implode and produce jetting and shock waves. Local effects that result from both types of cavitation include microstreaming and also the shear forces that cause membranes to form pores. Another bioeffect produced by focused US is sonoporation, the reversible permeabilisation of cell membranes to allow cellular uptake of molecules. This is usually facilitated by the presence of microbubbles (MBs) such as SonoVue (Bracco S.p.A, Milan, Italy). The pores formed due to the microbubble - cell interaction range from tens of nanometers ${ }^{10}$ to two micrometers ${ }^{11}$. Additional effects of focused US include increases in temperature. The effects produced by cavitation, sonoporation and heating can be used for tissue ablation, drug release from carriers and changes in cell membrane permeability $^{12}$.

UmTDD relies on transducers as the source of US. These are typically large and most commonly applied from outside the human body. They are coupled to the skin using US gel or water, and focused to an ellipsoidal region inside the body with dimensions of the order of $10 \mathrm{~mm}$ axially and $3 \mathrm{~mm}$ laterally. ${ }^{13}$ However, external application has limited usefulness for UmTDD as the US beam can be affected by media such as bone and gas, which can displace the beam and produce foci in the wrong place ${ }^{14}$. Furthermore, to ensure that the transducer remains focused at the desired target and to compensate for patient movement, complex arrangements are needed to continually readjust the focus. This involves combining US with a guidance system such as magnetic resonance imaging and increases the required staff skill and cost.

An approach that overcomes some of the difficulty associated with an external US transducer is to configure it intracorporeally. As we demonstrate here, this can be achieved by housing a focused US transducer in a capsule similar in size to a capsule endoscope device, which can then be applied for treatment throughout the length of the GI tract. This configuration avoids interference from bone and the need for the MRI guidance that is presently used: the focused ultrasound transducer is embedded in the capsule, which is inside the human body, overcoming the bone problem. The capsule also moves as the patient moves. Hence, these conventional reasons to use MRI no longer apply. Other advantages include the ability to use the capsule to monitor drug delivery, localise treatment, deliver and direct drugs towards the mucosa and promote uptake through the mucosa. One challenge for such intracorporeal transducers is the need to miniaturize the focused US transducer sufficiently to fit inside a capsule that remains ingestible, while retaining its ability to produce bioeffects that improve drug efficacy and uptake into the bowel wall. Another challenge is the need to include other components necessary for UmTDD in the limited space afforded by an ingestible capsule.

With this as the context, the aim of the work described here was the development of a proof-of-concept UmTDD capsule. The two main objectives were to determine the potential clinical usefulness of a miniature focused US transducer and to determine the feasibility of including it and other essential UmTDD components in an ingestible capsule. For proof-ofconcept, the capsule was designed to be tethered, to eliminate potential difficulties with power delivery. Positioning and localization were also absent from the design objectives; instead the efficacy of UmTDD in capsule form was addressed in detail. Nonetheless, discussion regarding potential solutions to these problems is included. Section 2 addresses the necessary aspects of capsule development and basic component validation and Section 3 presents preclinical results before conclusions are drawn in Section 4.

\section{Capsule Development}

This section describes the development of the capsule and necessary UmTDD components, including details of the fabrication and characterization of the focused US transducers.

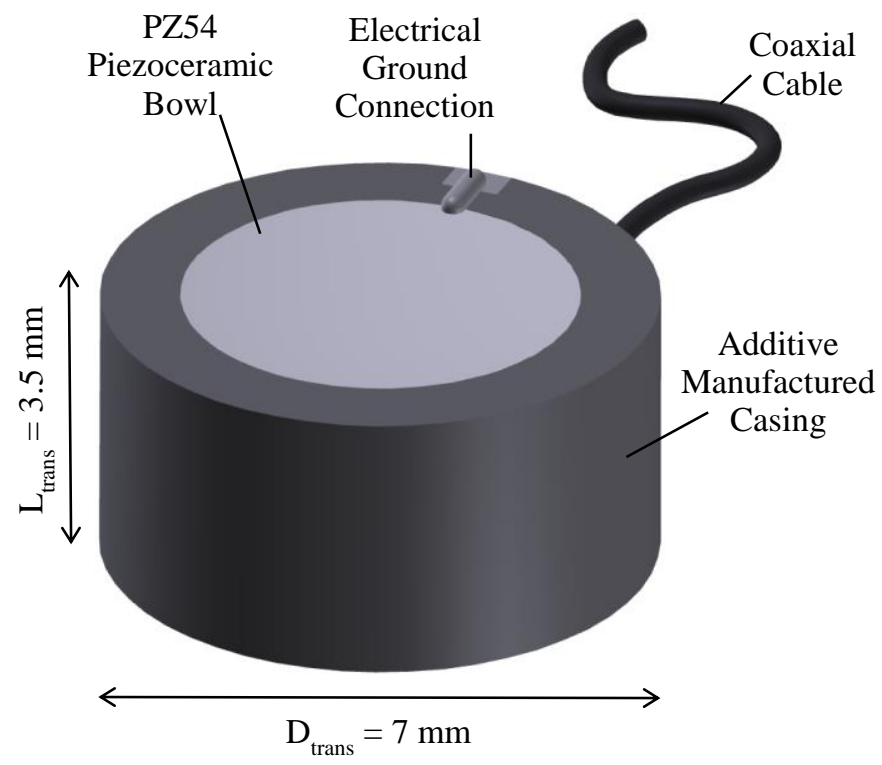

Fig. 1. Schematic of the focused US transducer comprising a PZ54 piezoceramic bowl, electrical connections to the front and rear faces of the piezoceramic, a coaxial cable with $\mathrm{OD}=0.3 \mathrm{~mm}$ and an additive manufactured transducer casing. A backing layer lies beneath the piezoelectric bowl. 


\subsection{Miniaturized Focused US Transducer Construction}

A conventional US transducer consists of an active piezoelectric element, an acoustic backing layer to damp reverberation, an acoustic matching layer to enhance energy transfer to and from the acoustic medium, electronic interconnects to external instrumentation and a protective casing ${ }^{12}$. However, bioeffects produced by focused US for improved drug uptake allow substantial simplification. This section describes the development of miniaturized focused US transducers that are suitable for this application.

US waves are produced by vibrations of a piezoelectric material in response to an applied AC voltage. In the present case, the US is focused to produce bioeffects in the focal region. Focusing the US beam can be achieved either by shaping the source geometrically or by beamforming with an US array. The latter allows electronic control of the beam direction and focal point location but involves great complexity in device fabrication and electronics. The former method was used in the present work, allowing an appropriately simple solution.

The focused US transducer must be small enough to be housed in an ingestible capsule with maximum diameter, $D_{c a p}=10 \mathrm{~mm}$, and length, $L_{c a p}=30 \mathrm{~mm}$, these dimensions are based on commercially available VCE devices. The piezoelectric component of choice was a PZ54 piezoceramic bowl (Meggitt Sensing Systems, Kvistgaard, Denmark) with outer diameter, $O D=5 \mathrm{~mm}$, radius of curvature, $R_{C}=15 \mathrm{~mm}$, thickness $T=0.5 \mathrm{~mm}$ and corresponding operating centre frequency, $f_{c}=4 \mathrm{MHz}$. PZ54 was developed specifically for focused US applications ${ }^{15}$; compared to other conventional piezoceramics such as PZ26 (Meggitt Sensing Systems, Kvistgaard, Denmark), it has higher permittivity and Curie temperature for better electrical impedance matching, with less variation in performance with temperature, allowing greater average US energy production.

A rendering of the fully fabricated transducer is shown in Fig. 1 and the fabrication process is described in detail in Fig. 2. The piezoceramic bowl is housed in a casing that provides structural support for the fragile piezoceramic and helps to form the backing layer. This was produced by additive manufacturing of ABS plastic using a Replicator 3D printer (MakerBot, New York City, USA). The casing diameter is $D_{\text {case }}=7 \mathrm{~mm}$, and length $L_{\text {case }}=3.5 \mathrm{~mm}$.

A reflective, low acoustic impedance backing layer was used to minimize energy absorption whilst providing physical support for the piezoceramic bowl. This comprised a mixture of K1 glass microbubbles (3M, Maplewood, MN, USA) and epoxy (Epofix, Struers A/S, Denmark) at a mass ratio of 3:1. This ratio forms a relatively solid material but with reduced density, thus lowering the acoustic impedance. The microbubble-loaded epoxy was applied to the rear surface of the bowl inside the case. After application, the transducer was placed in an oven to cure the backing layer at $70^{\circ} \mathrm{C}$ for $15 \mathrm{~min}$.

The silver electrodes as supplied on the PZ54 bowl were connected to a coaxial cable, $\varnothing=0.3 \mathrm{~mm}$, using conductive Agfilled epoxy (G3349, Agar Scientific, UK). This cable minimizes the transducer package size and the diameter of the capsule tether. The cable's inner conductor, $\varnothing=0.1 \mathrm{~mm}$, was connected to the rear surface of the transducer, consistent with safety. The outer screen was used for the ground connection, attached to the front surface of the transducer. The other end of the coaxial cable was terminated with an SMA connector.

\subsection{Ultrasound Transducer Characterization}

This section describes characterization of the focused US transducers. The output acoustic power and pressure were measured to demonstrate that sufficient energy can be generated for UmTDD.
A)

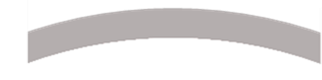

C)

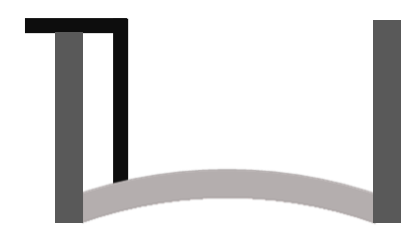

E)

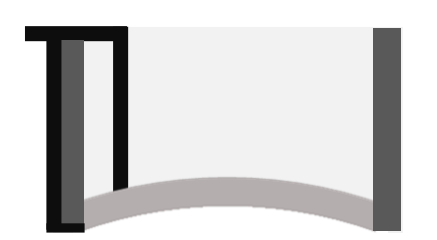

Piezoceramic

Backing Layer
B)

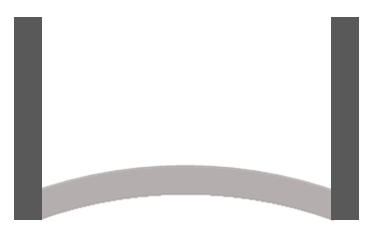

D)

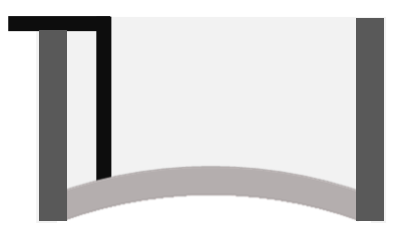

F)

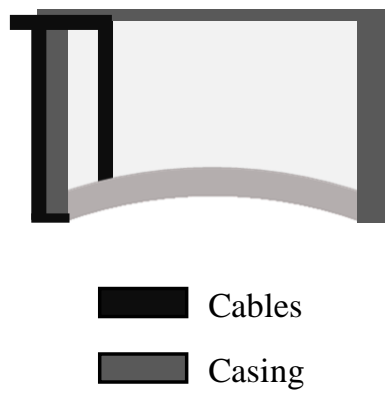

Fig. 2. Fabrication process for miniature focused US transducers. A) Piezoelectric material is placed face down on a glass plate. B) Additive manufactured case is placed over the piezoelectric material. C) The inner cable of the coaxial cable is attached to the rear surface of the piezoceramic using conductive silver epoxy. D) Glass-microbubble loaded epoxy is applied to the rear surface of the piezoceramic until it is flush with the casing. E) Electrical ground connection is attached to the side of the casing and connected to the front face of the transducer using conductive silver epoxy. F) The rear casing is attached and sealed with epoxy. 
The mechanisms of interaction between US and microbubbles are not completely understood yet but they are crucial for applications in clinical practice ${ }^{16}$. Furthermore, the literature still lacks standardized reporting of US parameters, including methods of exposure and the experimental conditions. This makes it difficult to compare existing results ${ }^{17,18}$ and means a definitive reference for our experiments is not available.

However, in general it has been found that drug uptake efficacy increases with US intensity and decreases with frequency ${ }^{19,20}$. Research significant for our study was conducted by Karshafian et al. ${ }^{19}$ who investigated systematically the effect of US exposure parameters on cell membrane permeability and correlated US-induced microbubble disruption with drug uptake efficacy. Qiu et al. also reported that acoustic pressures in the range $50-300 \mathrm{kPa}$ were suitable for reversible sonoporation without damage ${ }^{21}$.

\subsubsection{Acoustic Power Measurement}

Measuring acoustic power is one of the primary results in transducer characterization and provides an important quantitative description of output. This is also an important parameter to know to assess the ability of the fabricated transducer to disturb microbubbles in UmTDD. A radiation force balance (RFB) is the instrument that allows measuring these parameters in both diagnostic and therapeutic US. In an RFB the effect of US incident on an absorbing target causes displacement from the target's equilibrium position, which is then detected by a precision balance, and the magnitude of the equivalent mass is related directly to the radiation force.

A schematic diagram of the RFB with suspended target (Precision Acoustics, Dorchester, UK) is shown in Fig. 3. It can measure the total US power radiated in the forward direction from transducers operating in the frequency range $1-10 \mathrm{MHz}$.

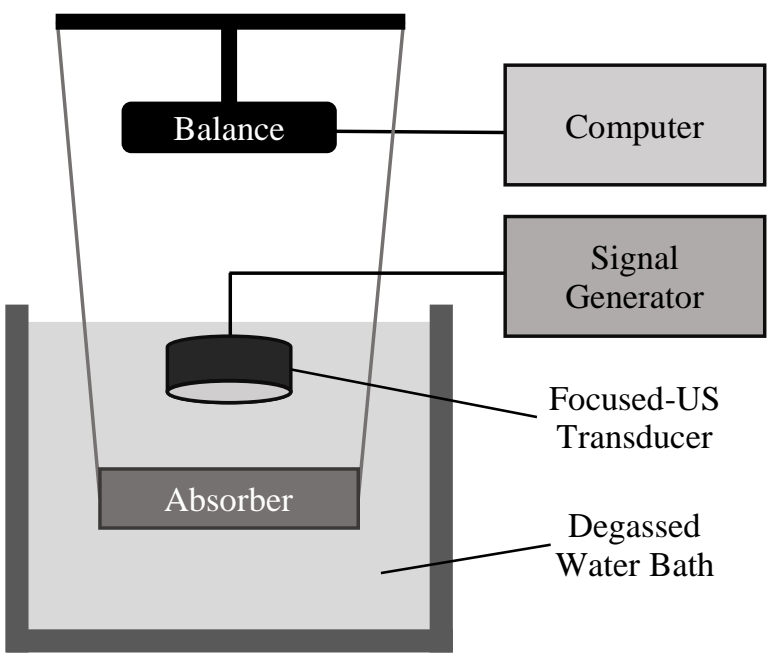

Fig. 3. Schematic diagram of a radiation force balance with suspended target. Focused-US transducer connected to a signal generator was placed in a degassed water bath with the focus at the absorber. The power was then measured from the change in mass on the balance, which is connected to a PC with dedicated software.

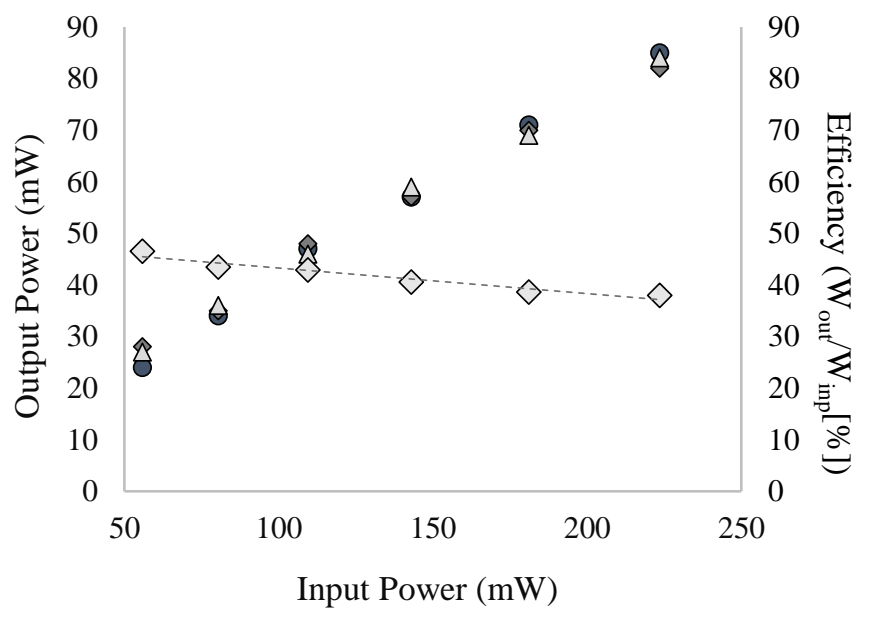

$\Delta \circ \diamond$ Output Power $(\mathrm{mW}) \quad \diamond$ Efficiency $(\%)$

Fig. 4. Linearity curve of the transducer: acoustic output power (mW) versus electrical input power $(\mathrm{mW})$. Efficiency (ratio between electrical input and acoustic output power [\%]) versus electrical input power $(\mathrm{mW})$.

The radiation force was then quantified as a change in mass using an analytical balance connected to a PC through a serial interface. The absorbing target was immersed in a water tank with the focused US transducer located above it and excited by a signal generator (Agilent 33220A Function/Arbitrary Waveform Generator). Dedicated LabView-based software (National Instruments, Texas, USA) controlled the RFB and signal source and provided data acquisition and automated analysis. Raw data refer to the registered mass values converted into power using a calibration factor predetermined for various temperatures.

The experiment was performed for different frequencies near the operating frequency of the transducer to obtain the value that maximized the output power. This frequency was subsequently used whilst the peak-to-peak voltage $\left(\mathrm{V}_{\mathrm{pp}}\right)$ input to the transducer was increased from $5-10 \mathrm{~V}_{\mathrm{pp}}$ with the US output power recorded. The resulting data were used to calculate output power from the transducer, and linearity and efficiency. The procedure was performed three times and averaged. For each specific input voltage, the US output power was recorded, and the averaged value was calculated.

The linearity and the efficiency of the transducer were calculated. The linearity can be obtained by comparing the acoustic output power measured by the RFB with the electrical input power driving the transducer. The electrical input power is calculated using Eq. (1).

$$
W_{\text {Input }}=\frac{V^{2} R M S}{Z}=\frac{\left(V_{p p} / 2 \sqrt{2}\right)^{2}}{Z}
$$

Where $V_{R M S}$ is the root mean square input voltage and $Z$ is the characteristic electrical impedance at the relevant frequency. 
The general linear relationship between $W_{\text {input }}$ and the averaged US output power are shown in Fig. 4. Additionally, the efficiency was calculated as the ratio between $W_{\text {input }}$ and the averaged US output power, also shown in Fig. 4. The effieciency varies between 38 and $47 \%$ and averages $42 \%$. At $8 V_{p p}$, this efficiency translates to power lost to heat of $85 \mathrm{~mW}$. Power lost to heat below $100 \mathrm{~mW}$ is safe as it ensures the capsule temperature remains below the safe limit of $43^{\circ} \mathrm{C}$ in vivo ${ }^{22}$.

\subsubsection{Acoustic Pressure Measurements}

The spatial distribution of the US field, including focal region, can be obtained using pressure mapping.

To map the pressure field at low intensities accurately, a needle hydrophone (Precision Acoustics, Dorchester, UK) with a submersible pre-amplifier and DC coupler was used. The hydrophone has a sensitive area of diameter, $\varnothing=0.075 \mathrm{~mm}$; the amplitude response is in the range $10-20 \mathrm{mV} / \mathrm{MPa}$ in the frequency range $1-30 \mathrm{MHz}$.

A commercial US Measurement System test tank (Precision Acoustics, Dorchester, England) was used for field mapping. It has a three-axis motorized step-and-repeat positioning frame covering a test tank filled with degassed water. A dedicated LabView-based software is provided with the system to control the positioning of the hydrophone within the tank. It also allows three different scan types, linear, planar and arbitrary. The working configuration of the system used in this study is shown in Fig. 5. The transducer was fixed to the tank with the active surface in the degassed water and a signal generator used for excitation. The hydrophone was mounted on the moving slide with the needle directed toward the transducer. The data were acquired and stored on a dedicated PC.

An oscilloscope (InfiniiVision 3024A, Agilent Technologies) was used to confirm in real time the input voltage and the US signal received. Pressure mapping requires a preliminary step to find the US focus in order to align the

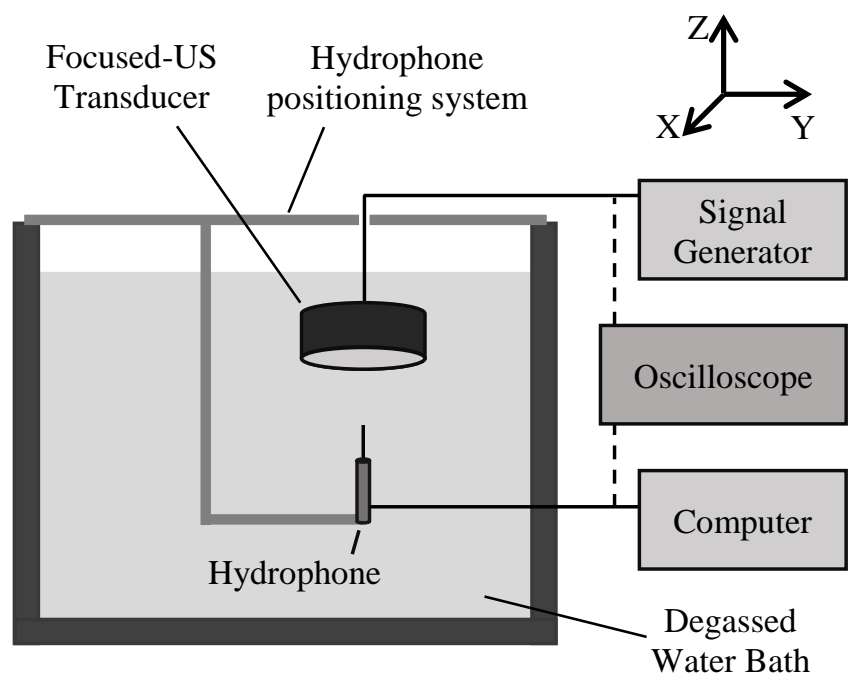

Fig. 5. Working configuration of system. The hydrophone is in the water tank, centred and facing the active surface of the transducer.

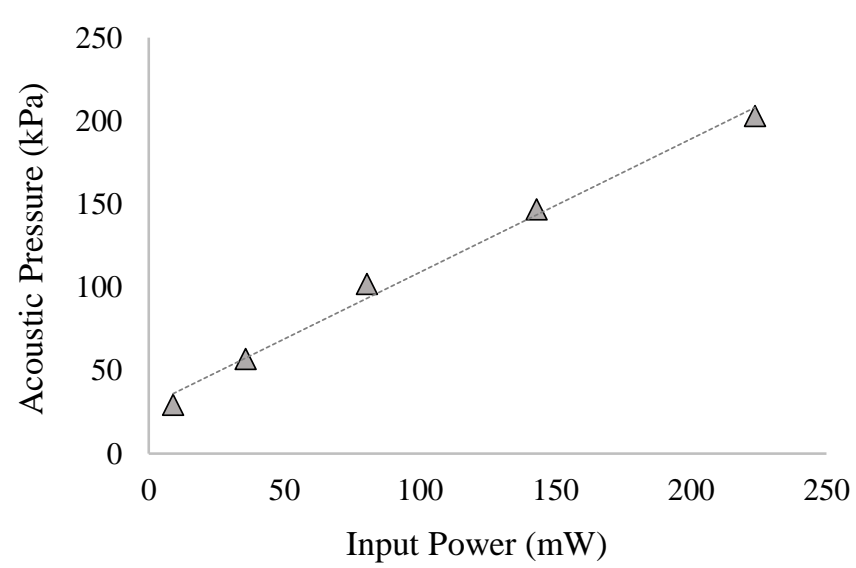

(a)

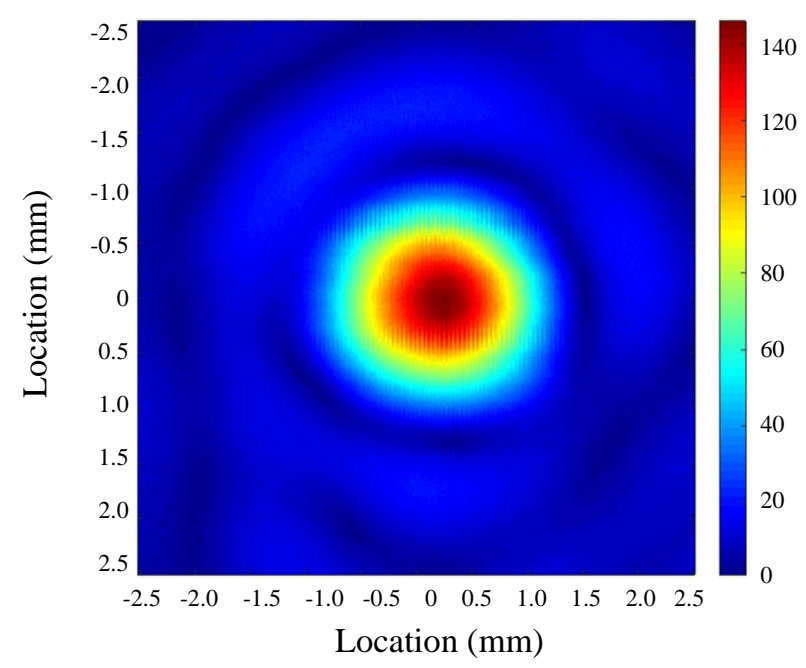

(b)

Fig. 6. (a): Acoustic output pressure ( $\mathrm{kPa}$ ) versus electrical input power (mW). (b): Representative cross-sectional scan of the pressure field at the transducer focal distance with an input power of $181 \mathrm{~mW}$.

hydrophone. After manually positioning the needle on the central axis of the transducer, single step movements were made along the main axis (refer to $\mathrm{z}$ axis in the coordinate system of Fig. 5). When a maximum in acoustic pressure was detected, an $\mathrm{x}-\mathrm{y}$ plane was then scanned to identify the focus coordinates.

Once the active surface of the hydrophone was positioned in at US focus, a planar scan was performed over $5 \mathrm{~mm} \times 5 \mathrm{~mm}$ with $0.075 \mathrm{~mm}$ steps. The displacement step should be smaller than the US wavelength, $\lambda=c / f$, where $c$ is the speed of sound in the water and $f$ the frequency. With $c=1500 \mathrm{~m} / \mathrm{s}$ and $f=4 \mathrm{MHz}$, the wavelength, $\lambda=0.6 \mathrm{~mm}$, is much larger than the step size. A continuous square wave was applied to the transducer at $\mathrm{f}=4 \mathrm{MHz}$, with the voltage varied from $2-10 \mathrm{~V}_{\mathrm{pp}}$ in increments of $2 \mathrm{~V}_{\mathrm{pp}}$. Data was post-processed using both the post processing software provided with the system and MATLAB. The hydrophone sensitivity, i.e. the relationship 
between input pressure and output voltage was $18 \mathrm{mV} / \mathrm{MPa}$ at $4 \mathrm{MHz}$; this was used to convert the acquired voltage data into pressure information.

The pressure results are shown in Fig. 6. The output acoustic pressure was measured from $9-224 \mathrm{~mW}$ generating pressures in the range $29.5 \mathrm{kPa}<p_{a c}<208.1 \mathrm{kPa}$. With an input power of $181 \mathrm{~mW}$, corresponding to $p_{a c}=147 \mathrm{kPa}$, there is a beam diameter of $1.5 \mathrm{~mm}$ at $-6 \mathrm{~dB}$.

\subsection{Capsule Design}

The system that was developed comprises an insonation capsule for autonomous imaging and therapy (SonoCAIT) and a soft tether providing electrical connections and other channels. At the present stage in proof-of-concept development, wireless communications were neglected, however they will be addressed in future iterations. SonoCAIT has a pill-like shape with the dimensions of a commercial capsule (PillCam Colon, Given Imaging Ltd., Yoqneam, Israel) noted previously. Fig. 7. (a) shows the $3 \mathrm{D}$ computer-aided design model of the first prototype, designed with SolidWorks (Dassault Systèmes SOLIDWORKS Corp. Waltham, MA). In Fig. 7. (b) the first additively manufactured capsule is shown; it was printed in two halves which lock together using an Objet Connex 500 printer (Stratasys Ltd., Minnesota, USA).

The housing for the capsule components, i.e. the transducer, drug delivery channel and vision module, and the multi-channel external tether are also indicated. The focused US transducer is as described previously. The vision module comprises a CMOS camera and an LED-based illumination board. The camera (microScoutCam ${ }^{\mathrm{TM}}$, Medigus Ltd., Israel) is cylindrical, $\varnothing$ $1.2 \mathrm{~mm}$, length $5 \mathrm{~mm}$, with an image area of $492.8 \mu \mathrm{m} \times 488.4$ $\mu \mathrm{m}$ and a resolution of $220 \times 224$ pixels. It is connected to a matching video processor which captures and saves images and video. The LED-based illumination comprised a printed circuit board with four $40 \mathrm{~mW}$ white LEDs (OSRAM Opto Semiconductors GmbH, Germany) placed at the distal tip of the camera.

The multi-channel external tether is also shown in Fig. 7. (a). This has an outer diameter of $2.25 \mathrm{~mm}$ and an inner diameter

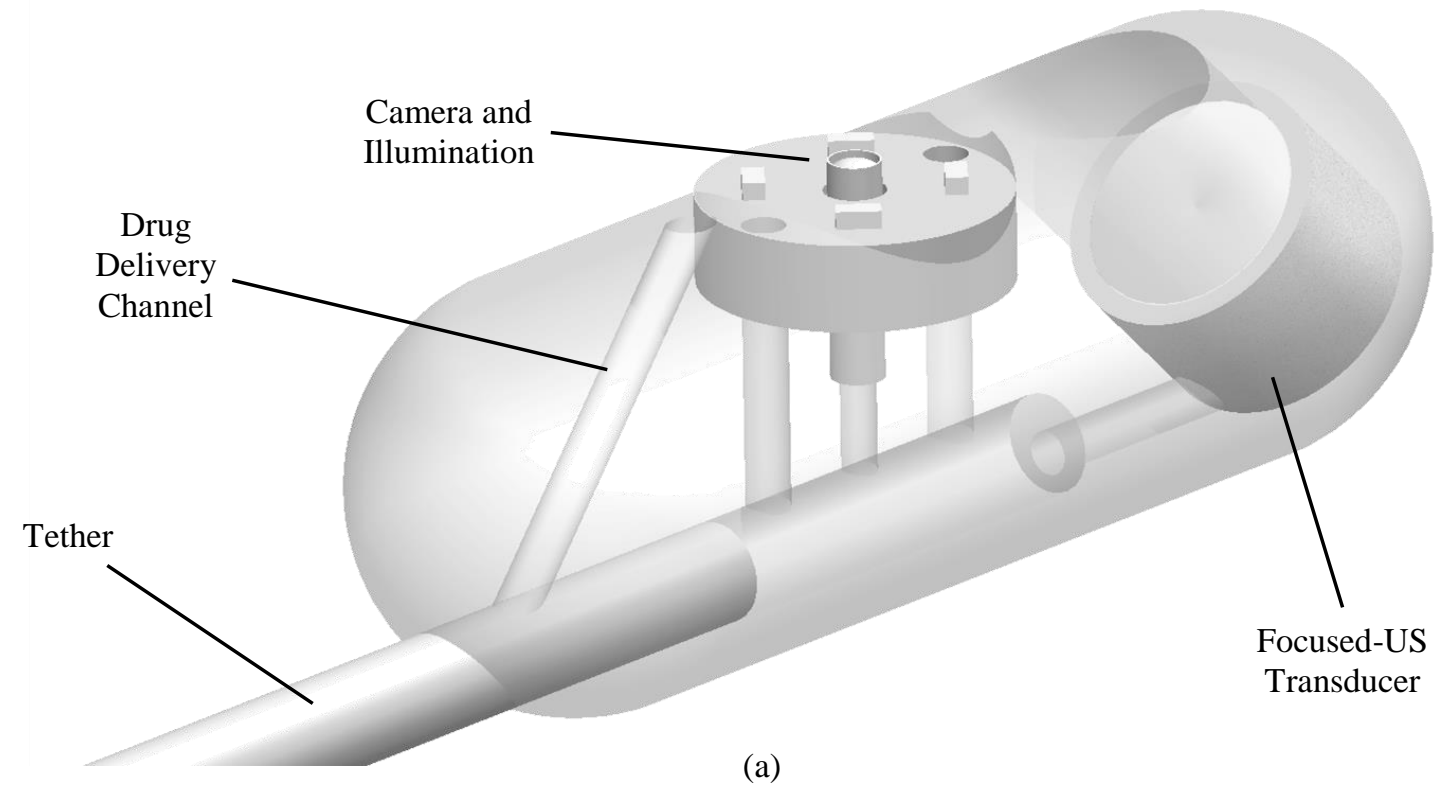

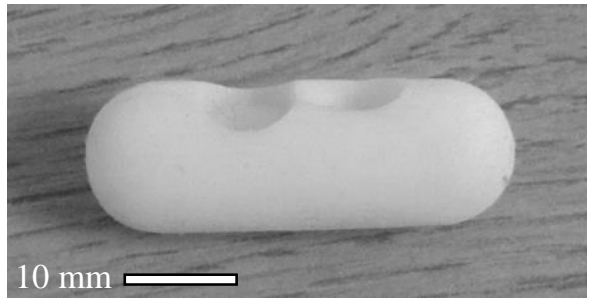

(b)

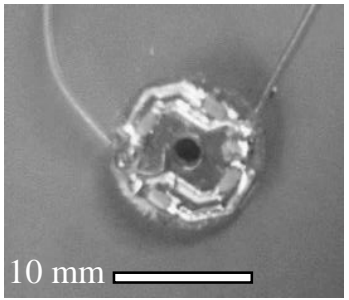

(c)

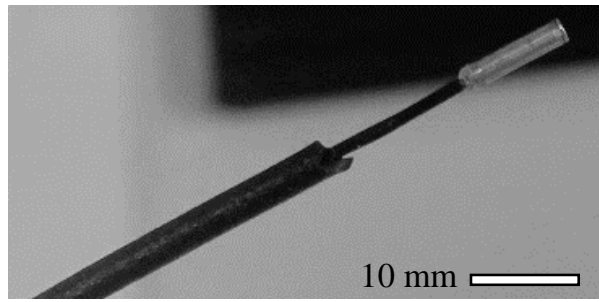

(d)

Fig. 7. (a): Computer-assisted drawing of the constructed SonoCAIT with components labelled (NB all wiring omitted for clarity). (b): Capsule shell produced by additive manufacturing measuring $10 \mathrm{~mm}$ in diameter and $30 \mathrm{~mm}$ in length. (c): Illumination printed circuit board with 4 LEDs in an annular pattern. (d): Miniature CMOS imaging camera, measuring $1.2 \mathrm{~mm}$ in diameter and $5 \mathrm{~mm}$ in length. 
of $1.65 \mathrm{~mm}$. The tether houses the power cables for the camera and illumination and the signal excitation cable for the transducer, along with the drug delivery channel. The latter is fine bore polythene tubing with outer diameter $0.96 \mathrm{~mm}$ and inner diameter $0.58 \mathrm{~mm}$. The tether connects the capsule components with the bench-top apparatus for camera/transducer control and drug delivery. As a repurposed vascular catheter, the tether sheath was designed such that $360^{\circ}$ rotation at the proximal end results in the same rotation at the distal end which can be exploited for positioning the capsule toward the target location.

The aim of SonoCAIT is to deliver drugs to the wall of the GI tract. One example of a therapeutic preparation are drugfilled microbubbles. When these reach the target zone, they must be released in close proximity to the wall where the drugs can then be released by US. ${ }^{23}$ That means the US focus and microbubbles have to be directed towards the same target. Clinical measurements suggest that the size of the lumen of the bowel means a suitable distance from the capsule perimeter to the target zone is $5 \mathrm{~mm}$. Correspondingly, the capsule was designed with the transducer, camera and drug delivery channel housed at angles such that they are confocal at this distance, Fig. 7 (a).

\section{Capsule Feasibility Testing}

The potential clinical usefulness of SonoCAIT and its application in UmTDD was demonstrated experimentally. First, two functional tests were set up: A) optical tests to monitor camera positioning in the capsule and B) feasibility tests to determine miniaturized focused US effects on microbubbles. Second, in vitro testing was performed with a small bowel epithelium model.

\subsection{Primary Functional Testing}

The arrangements for the optical and focused US feasibility experiments included a signal generator to drive the transducer, a syringe pump connected to the drug delivery channel, a DC power supply for video illumination and the video processor for

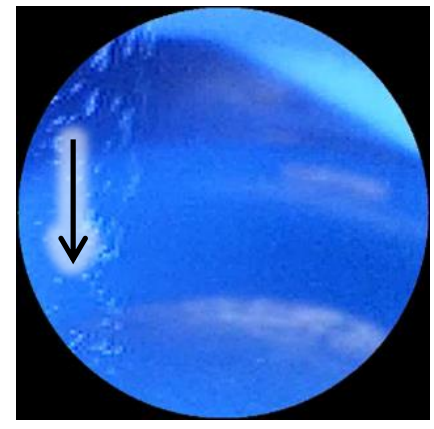

(a)

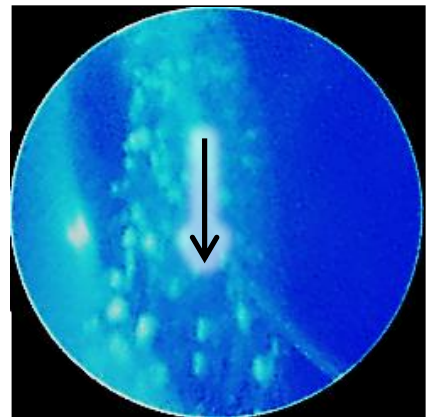

(b)
Fig. 8. (a): Initial result before alignment. Microbubbles were clearly off centre as indicated by arrow. (b): After camera and delivery channel adjustment, the microbubble stream was central to the FoV.

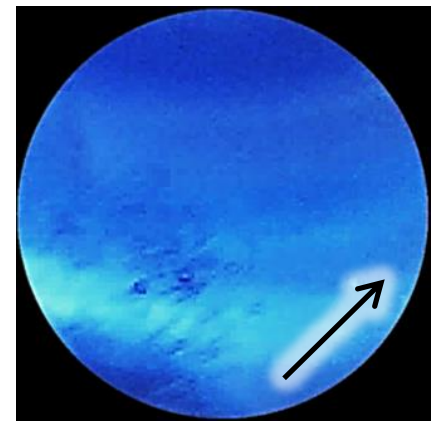

(a)

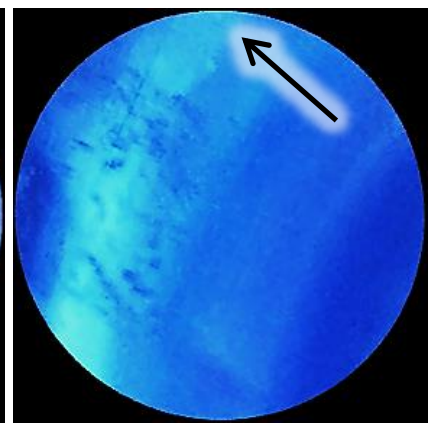

(b)
Fig. 9. (a): MB stream leaving the drug delivery channel and approaching the US focus. (b): MBs after changing direction due to the acoustic radiation forces exerted at the US focus.

the camera. During the experiments, SonoCAIT was immersed in a glass beaker of degassed water.

\subsubsection{Optical Assessment}

The aim of this experiment was to verify the orientation and alignment of the components enclosed in SonoCAIT. Alignment is particularly important because the camera may not be able to record the regions of interest and/or the US beam may not reach the target with the correct intensity if these components are misaligned.

To align the camera, $\varnothing 65 \mu \mathrm{m}$ microbubbles (3M, Maplewood, MN, USA) mixed with degassed water were passed through the DDchannel using the syringe pump (BRAUN perfusor FM Pump IV Infusion syringe pump B, Braun Medical Ltd., Sheffield, UK) and monitored with the camera. The goal of this test was to confirm that the camera could detect the bubble stream and to ensure that the MBs were central in its field of view (FoV).

Microbubbles were passed through the drug delivery channel as described above and images were acquired, Fig. 8 (a). Initially the MB stream was off centre from the camera FoV. This was corrected by repositioning the camera within the capsule with subsequent images showing the MB stream in the centre of the FoV, Fig. 8 (b), confirming that the camera and DD pathway converged at the same point.

\subsubsection{Focused US Effect on Microbubbles}

The arrangements to characterize the microbubble behavior at the focus of the transducer were the same as above. Microbubbles were passed through the drug delivery channel and the US transducer was driven at $181 \mathrm{~mW}$, corresponding to an acoustic pressure of $147 \mathrm{kPa}$ and acoustic output power of $58.1 \mathrm{~mW}$, at $\mathrm{f}=4 \mathrm{MHz}$. The capsule was designed such that the radiation force should push the MB stream towards the bowel wall to facilitate drug uptake.

Housing the US transducer within SonoCAIT together with the camera, illumination and drug delivery channel revealed the ability of acoustic radiation forces to influence MBs. Fig. 9 (a) shows the MB stream approaching the US focus with a velocity 
of $0.82 \mathrm{~mm} / \mathrm{s}$ and Fig. 9 (b) shows a change in its direction by approximately $90^{\circ}$ due to acoustic radiation force generated when the transducer is excited, with a final velocity of $0.45 \mathrm{~mm} / \mathrm{s}$. The superimposed arrows in Fig. 9 (a) and (b) indicated the directions of the stream. Drugs are frequently coupled with MBs for UmTDD, either by containing the drug inside the MB or by attaching it to the MB shell12, 24. Therefore, our system can redirect the drug - MB combination towards the target site (i.e. towards the transducer focus), with the consequent capability to enhance drug efficacy. Supplementary video 1 shows the MB redirection in real time. Importantly, the ability of the US in the capsule to redirect the MB shows that focused-US transducer, drug delivery and camera converged to the same point.

\subsection{In Vitro Study of Miniaturized Focused US Effects}

To determine the potential biological efficacy of focused US in miniaturized capsule form, in vitro tests were performed using cultured human colon cancer (Caco-2) cells. Once differentiated and polarised, Caco- 2 cells mimic the enterocytes lining the small intestine ${ }^{25}$, forming cellular junctions and apical microvilli. Enzymes and transporters present in the Caco- 2 cells reflect those found in the small intestine epithelium. Such differentiated Caco-2 cells are approved by the Food and Drug Administration as a model of the small intestine ${ }^{26}$.

\subsubsection{Cell Culture}

Caco- 2 cells were maintained in DMEM (Thermo Fisher Scientific, Waltham, MA, USA) supplemented with $10 \%$ FBS (GE Life Sciences, Chicago, USA), 1\% NEAA (Thermo Fisher Scientific, Waltham, MA, USA) and $0.5 \%$ Penicillin Streptomycin (Thermo Fisher Scientific, Waltham, MA, USA) under sterile conditions. Cells were seeded on ThinCert suspended membranes (Greiner Bio-One, Kremsmunster, Austria) at a density of 500,000 cells per 12-well filter. Transepithelial electrical resistance (TER) measurements were

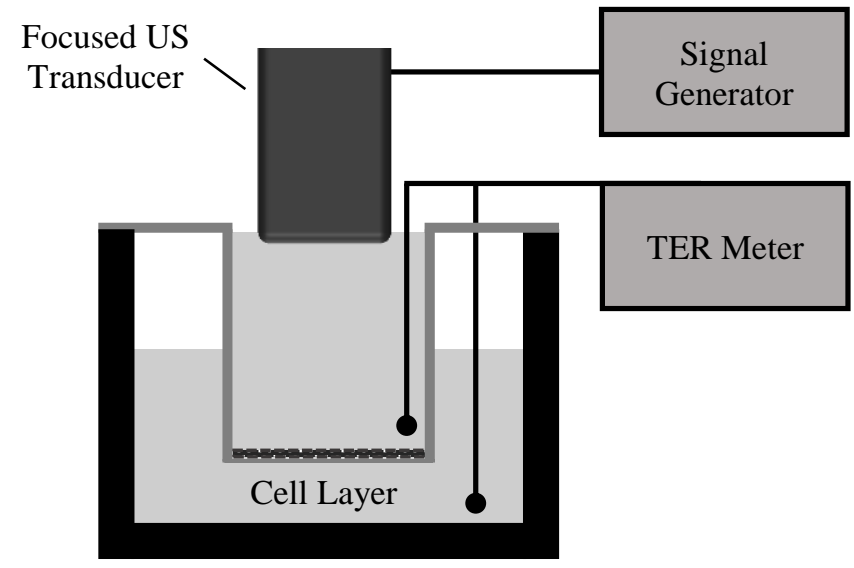

Fig. 10. Set up showing location of focused US transducer and TER probe in relation to cell layer. The focused US transducer is driven by a waveform generator. The TER probe measures the resistance across the cell layer and sends the reading to the TER meter.

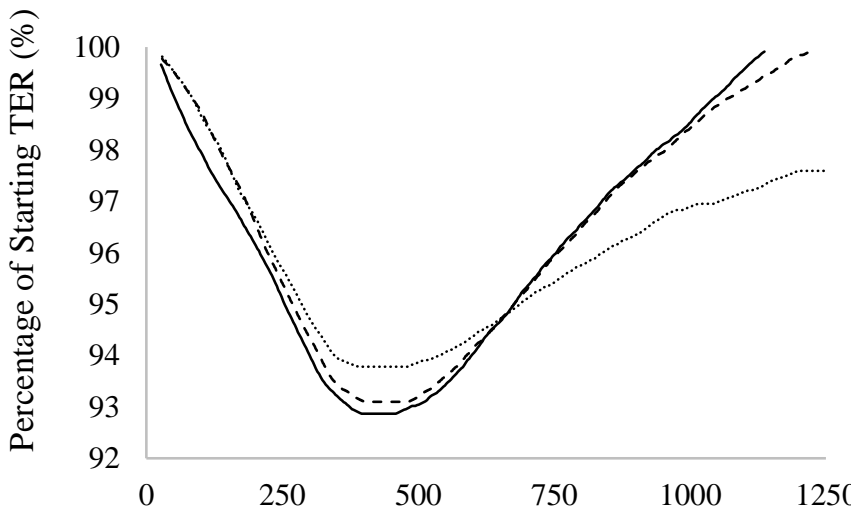

Time (s)

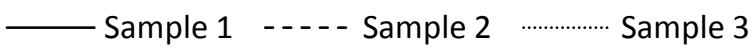

Fig. 11. TER (shown as percentage of starting value) versus time for three samples. The insonation time for each sample was 6 minutes. For all samples, there is a drop of $6-7 \%$ after $t=6$ mins before the TER plateaus and begins to recover.

recorded every three days using a Millicell-ERS meter (Millipore, Billerica, MA, USA) to assess barrier function of the epithelial layer. TER was calculated using Eq. (2).

$$
T E R=\left(R-R_{\text {control }}\right) * A_{\text {membrane }}
$$

Where $R$ is the resistance read from the meter, $R_{\text {control }}$ is the resistance across a membrane with no cells present and $A_{\text {membrane }}$ is the area of the membrane on which the cells are grown. After 21 - 25 days, Caco- 2 cells represent an accepted model of the small intestine, attaining a TER in the range $500-1000 \Omega \mathrm{cm}^{2}$, 27,28 .

\subsubsection{Focused US Effect of TER}

Cells were sonicated with a focused US insonation system ${ }^{29}$ with exactly the same US transducer design and excitation system as in SonoCAIT. Only the casing and connector were altered to allow interchangeability between transducers and sterilization.

To measure TER during sonication, Caco- 2 cells grown on ThinCerts as described in Section 3.2.1 were transferred to the insonation system and the focused US transducer and TER probe were inserted into the well (Fig. 10). A continuous, sinusoidal waveform was applied to the transducer with an input power of $181 \mathrm{~mW}$, corresponding to an acoustic pressure $p_{\mathrm{ac}}=$ $147 \mathrm{kPa}$ and acoustic power $W_{\text {out }}=58.1 \mathrm{~mW}$. Focused US was applied for time $\mathrm{t}=6$ minutes.

The original resistance decreased between $6-7 \%$ during the insonation in all three cases. After insonation (Figure 11). TER recovered to its initial value after $11-12$ mins in Samples 1 and 2. However, in sample three it recovered to only $97.6 \%$ of its initial value and did so more slowly. The reason for the difference in Sample 3 is not known. Variability in cell packing 
is a possible reason but is unlikely as cells in all three samples were seeded at identical densities and the prolonged growth period before measurements were taken should ensure even distribution in all samples.

TER is a measure of the barrier function of cells ${ }^{28,30}$ Therefore, the drop in TER during insonation is an indication of decreased barrier function in response to US. A temporary release of intercellular junctions, such as tight junctions, or an increase in cell membrane permeability could be responsible. Either of these effects could increase drug uptake. That means, using the miniature focused US transducers at relatively low excitation voltage could facilitate drug uptake.

\section{Conclusions}

This paper presents a proof-of-concept device for TCE that could ultimately be applied as an UmTDD system in the GI tract.

The capsule included a bespoke, miniature focused US transducer prepared specifically for this purpose in-house. A vision module including illumination and miniature CMOS imaging camera were sourced commercially and a drug delivery channel was set up. PZ54 (Meggitt Sensing Systems, Kvistgaard, Denmark) was chosen as the transducer material for its combination of high piezoelectric activity and high permittivity resulting in an improved power level for a small size. The vision module comprised a Micro ScoutCam 1.2 (Medigus, Yoqneam, Israel) small enough to fit into the capsule and custom-designed LED illumination. The drug delivery channel was fashioned from fine bore polythene tubing and delivery was achieved with an external syringe pump. These components were assembled in a shell made with additive manufacturing with a repurposed intravascular catheter acting as a tether. The focused US transducer exhibited good linearity and adequate efficiency, and provided a maximum acoustic power of $58.1 \mathrm{~mW}$ and acoustic pressure of $146 \mathrm{kPa}$ with $8 \mathrm{~V}_{\mathrm{pp}}$ excitation, making it suitable for reversible sonoporation as described by Qiu et al. ${ }^{21}$ The camera, drug delivery channel and focused US transducer were arranged confocally.

Feasibility tests included passing MBs through the drug delivery channel and monitoring the behavior at the US focus. We found that the acoustic radiation force from the transducer could redirect the MB stream by approximately $90^{\circ}$; however substantive quantitative data is still to be obtained. This shows that the miniature transducers can direct MBs towards the target site, i.e. the transducer focus. Since MBs are often coupled with drugs in UmTDD applications ${ }^{24}$, this has the potential to increase the amount of drug delivered to the target site, which could improve treatment. The focused US transducer configuration was also tested in vitro using an insonation system that matched that used in the capsule. Caco-2 cells grown for 21 - 25 days on ThinCerts (Greiner Bio-One, Kremsmunster, Austria) were used as a model of the small intestine, when their TER reached $500-1000 \Omega \mathrm{cm}^{2}$. TER was measured during insonation to investigate the effect of US on barrier function. A drop in TER of $6-7 \%$ occurred during insonation with recovery to near starting values within $11-12$ minutes. The drop in TER likely indicates a change in tight junctions and/or to an increase in cell membrane permeability. Further experiments will be carried out to determine the enhanced drug uptake to which this drop in TER corresponds. Both MB and TER experiments were carried out in liquids used in vitro, thus providing coupling for the US to the tissue model itself. In vivo, there may be liquidgas interfaces that could disrupt the US path, although this is not the authors experience in tests in a large animal model in vivo. It is unclear how much of an effect this may have but further investigation remains necessary ${ }^{22}$.

Furthermore, we show that SonoCAIT transducers are small enough to fit in an ingestible capsule and can produce clinically useful effects while maintaining an achievable power demand of less than $230 \mathrm{~mW}$. In contrast, focused US transducers currently used for non-capsule based UmTDD have power demands in excess of $100 \mathrm{~W}$, which is unachievable in capsule form.

Presently, the prototype device is tethered to allow bench testing and feasibility studies to be performed. Future iterations of the device will be wireless to allow autonomous therapy in the GI tract. One means to achieve this which has been explored by researchers collaborating with the authors is by utilizing an antenna embedded in the outer casing of the capsule ${ }^{31}$. This configuration optimizes space within the capsule whilst allowing power to be transmitted to and data to and from the capsule. Elsewhere, the authors have explained how the present therapeutic capsule may be used in conjunction with a diagnostic capsule ${ }^{32}$ to allow both diagnosis and therapy to be accommodated within the same capsule volume as present VCE devices. This partitions the system to allow space for the key therapeutic functions described here whilst minimizing the diagnostic functions required of the therapeutic capsule.

Positioning and localization functionality was also excluded in this study as it was specifically designed to elucidate the feasibility of UmTDD as a therapeutic modality for capsule endoscopy. However, future iterations of the capsule will include positioning and localization functions to enhance treatment efficacy. Attempts have been made previously to combine positioning with capsule endoscopy: Quirini et al. used leg-based locomotion to propel the capsule ${ }^{33}$; Woo et al. proposed that electrical stimulation of the GI muscle could provide capsule locomotion ${ }^{34}$; and Valdastri et al. developed a capsule that used an external magnet controlled by a robotic arm to propel a capsule containing an internal magnet through the GI $\operatorname{tract}^{35}$.

Presently, the most suitable positioning modality for a therapeutic capsule is a system similar to that developed by Valdastri et al, making use of external and internal magnets. This is because their system is able to move the capsule arbitrarily forwards and backwards and to anchor it in place, vital functions for UmTDD. In addition, the system by Valdastri et al also provides localization through magnetic sensors in the capsule. This is necessary to allow diseased regions to be identified after diagnosis and to provide feedback to the positioning system. Furthermore, magnetic coupling will provide capsule contact with the GI wall, necessary for US transmission but also creating a problem with a camera that has 
a focal distance of $5 \mathrm{~mm}$ and is currently situated at the surface of the capsule. Since a system similar to that developed by Valdastri et al. requires internal magnets embedded in the capsule, a capsule redesign would be necessary to incorporate all components. During the redesign, the camera could be offset by $5 \mathrm{~mm}$ from the capsule surface, allowing it to focus on the bowel wall.

In summary, our proof-of-concept study revealed SonoCAIT as a potentially useful clinical tool. The transducer could direct a MB stream with acoustic radiation forces and reduced TER across an in vitro model of the gut wall. The transducer produced acoustic pressure in the range suitable for reversible sonoporation. Extending these results to a clinical device could allow drug-filled MBs to be pushed towards the wall of the intestine where they would release their contents under the influence of US that also simultaneously could increase drug uptake. Adding robotic functionality to assist localization and positioning would greatly enhance the usefulness of the SonoCAIT in localised treatment of GI disorders such as Crohn's disease, which is presently treated systemically with drugs that have unwanted side effects and may be highly toxic.

\section{Acknowledgments}

Financial support from the UK Engineering and Physical Sciences Research Council, Grant EP/K034537 (Sonopill Programme) is gratefully acknowledged.

The authors would also like to thank Dr Maya Thanou from King's College London for many helpful discussions.

\section{References}

[1] Kaplan, G.G. (2015) The global burden of IBD: from 2015 to 2025. Nature Reviews Gastroenterology \& Hepatology 12, 720727.

[2] Riccioni, M.E., Urgesi, R., Cianci, R., Bizzotto, A., Spada, C., and Costamagna, G. (2012) Colon capsule endoscopy: Advantages, limitations and expectations. Which novelties. World J Gastrointest Endosc 4, 99-107.

[3] Koprowski, R. (2015) Overview of technical solutions and assessment of clinical usefulness of capsule endoscopy. Biomedical engineering online 14, 111.

[4] Cummins, G., Lay, H., Cox, B., Seetohul, V., Qiu, Y., Stewart, F., Faerber, J., Mitrakos, V., Al Rawhani, M., and Beeley, J. Sonopill: A Platform for Gastrointestinal Disease Diagnosis and Therapeutics.

[5] Wilding, I., Hirst, P., and Connor, A. (2000) Development of a new engineering-based capsule for human drug absorption studies. Pharmaceutical science \& technology today 3, 385-392.

[6] Parr, A.F., Sandefer, E.P., Wissel, P., McCartney, M., McClain, C., Ryo, U., and Digenis, G.A. (1999) Evaluation of the feasibility and use of a prototype remote drug delivery capsule (RDDC) for non-invasive regional drug absorption studies in the GI tract of man and beagle dog. Pharmaceutical research 16, 266-271.

[7] Yim, S., Goyal, K., and Sitti, M. (2013) Magnetically actuated soft capsule with the multimodal drug release function. IEEE/ASME Transactions on Mechatronics 18, 1413-1418.
[8] Xitian, P., Hongying, L., Kang, W., Yulin, L., Xiaolin, Z., and Zhiyu, W. (2009) A novel remote controlled capsule for sitespecific drug delivery in human GI tract. International journal of pharmaceutics 382, 160-164.

[9] Woods, S.P., and Constandinou, T.G. (2013) Wireless capsule endoscope for targeted drug delivery: mechanics and design considerations. IEEE Transactions on Biomedical Engineering 60, 945-953.

[10] Lentacker, I., De Cock, I., Deckers, R., De Smedt, S., and Moonen, C. (2014) Understanding ultrasound induced sonoporation: definitions and underlying mechanisms. Advanced drug delivery reviews 72, 49-64.

[11] Zhao, Y.-Z., Luo, Y.-K., Lu, C.-T., Xu, J.-F., Tang, J., Zhang, M., Zhang, Y., and Liang, H.-D. (2008) Phospholipids-based microbubbles sonoporation pore size and reseal of cell membrane cultured in vitro. Journal of drug targeting 16, 18-25.

[12] Hernot, S., and Klibanov, A.L. (2008) Microbubbles in ultrasound-triggered drug and gene delivery. Advanced drug delivery reviews 60, 1153-1166.

[13] Chen, H., and Hwang, J.H. (2013) Ultrasound-targeted microbubble destruction for chemotherapeutic drug delivery to solid tumors. Journal of therapeutic ultrasound 1, 10.

[14] Staruch, R.M. (2013) Hyperthermia Mediated Drug Delivery using Thermosensitive Liposomes and MRI-Controlled Focused Ultrasound. University of Toronto.

[15] Qiu, Z. (2014) Development of MRI-compatible transducer array for focused ultrasound surgery: the use of relaxor-based piezocrystals. University of Dundee.

[16] Yu, T., Wang, Z., and Mason, T.J. (2004) A review of research into the uses of low level ultrasound in cancer therapy. Ultrasonics sonochemistry 11, 95-103.

[17] Kinoshita, M., and Hynynen, K. (2007) Key factors that affect sonoporation efficiency in in vitro settings: the importance of standing wave in sonoporation. Biochemical and biophysical research communications $359,860-865$.

[18] Civale, J., Rivens, I., and ter Haar, G. (2015) Quality assurance for clinical high intensity focused ultrasound fields. International Journal of Hyperthermia 31, 193-202.

[19] Karshafian, R., Bevan, P.D., Williams, R., Samac, S., and Burns, P.N. (2009) Sonoporation by ultrasound-activated microbubble contrast agents: effect of acoustic exposure parameters on cell membrane permeability and cell viability. Ultrasound in medicine \& biology 35, 847-860.

[20] Meijering, B.D., Henning, R.H., Van Gilst, W.H., Gavrilović, I., Van Wamel, A., and Deelman, L.E. (2007) Optimization of ultrasound and microbubbles targeted gene delivery to cultured primary endothelial cells. Journal of drug targeting 15, 664-671.

[21] Qiu, Y., Zhang, C., Tu, J., and Zhang, D. (2012) Microbubbleinduced sonoporation involved in ultrasound-mediated DNA transfection in vitro at low acoustic pressures. Journal of biomechanics 45, 1339-1345.

[22] Lay, H., Cummins, G., Cox, B., Qiu, Y., Connor, C., Seetohul, V., Turcanu, M., McPhillips, R., Desmulliez, M., Clutton, E., and Cochran, S. (2017) Translational Trial Outcomes for Capsule Endoscopy Test Devices. Ultrasonics Symposium (IUS), 2017 IEEE International, 1-4.

[23] Kilroy, J.P., Patil, A.V., and Hossack, J.A. (2009) Ultrasound catheter for microbubble based drug delivery. Ultrasonics Symposium (IUS), 2009 IEEE International, 2770-2773.

[24] Klibanov, A.L., Shevchenko, T.I., Raju, B.I., Seip, R., and Chin, C.T. (2010) Ultrasound-triggered release of materials entrapped 
in microbubble-liposome constructs: a tool for targeted drug delivery. Journal of Controlled Release 148, 13-17.

[25] Meunier, V., Bourrie, M., Berger, Y., and Fabre, G. (1995) The human intestinal epithelial cell line Caco-2; pharmacological and pharmacokinetic applications. Cell biology and toxicology 11, 187-194.

[26] Administration, F.a.D. (2000) Guidance for industry: Waiver of in vivo bioavailability and bioequivalence studies for immediate relase solid oral dosage forms based on a biopharmaceutics classification system.

[27] Srinivasan, B., Kolli, A.R., Esch, M.B., Abaci, H.E., Shuler, M.L., and Hickman, J.J. (2015) TEER measurement techniques for in vitro barrier model systems. Journal of laboratory automation 20, 107-126.

[28] Chen, S., Einspanier, R., and Schoen, J. (2015) Transepithelial electrical resistance (TEER): a functional parameter to monitor the quality of oviduct epithelial cells cultured on filter supports. Histochemistry and cell biology 144, 509-515.

[29] Stewart, F., Cox, B., Wang, G., Huang, Z., Newton, I., Nathke, I., Thanou, M., and Cochran, S. (2016) An in vitro sonication system for applications in ultrasound-mediated targeted drug delivery. Ultrasonics Symposium (IUS), 2016 IEEE International, 1-4.

[30] Klingberg, T.D., Pedersen, M.H., Cencic, A., and Budde, B.B. (2005) Application of measurements of transepithelial electrical resistance of intestinal epithelial cell monolayers to evaluate probiotic activity. Applied and environmental microbiology 71, 7528-7530.

[31] Faerber, J., Cummins, G., and Desmulliez, M.P. (2016) Design of conformai wideband antennas for capsule endoscopy within a body tissue environment. Microwave Conference (EuMC), 2016 46th European, 1223-1226.

[32] Stewart, F.R., Qiu, Y., Lay, H.S., Newton, I.P., Cox, B.F., AlRawhani, M.A., Beeley, J., Liu, Y., Huang, Z., and Cumming, D.R. (2017) Acoustic Sensing and Ultrasonic Drug Delivery in Multimodal Theranostic Capsule Endoscopy. Sensors 17, 1553.

[33] Quirini, M., Scapellato, S., Valdastri, P., Menciassi, A., and Dario, P. (2007) An approach to capsular endoscopy with active motion. Engineering in Medicine and Biology Society, 2007. EMBS 2007. 29th Annual International Conference of the IEEE, 2827-2830.

[34] Woo, S.H., Kim, T.W., Mohy-Ud-Din, Z., Park, I.Y., and Cho, J.-H. (2011) Small intestinal model for electrically propelled capsule endoscopy. Biomedical engineering online 10, 108.

[35] Valdastri, P., Ciuti, G., Verbeni, A., Menciassi, A., Dario, P., Arezzo, A., and Morino, M. (2012) Magnetic air capsule robotic system: proof of concept of a novel approach for painless colonoscopy. Surgical endoscopy 26, 1238-1246.

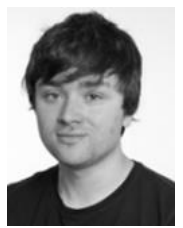

Fraser Stewart received his BSc degree from the University of Dundee in 2014. He joined the Sonopill team in 2014 as a $\mathrm{PhD}$ student investigating the feasibility of ultrasoundmediated targeted drug delivery in capsule form.

His main research interests include therapeutic ultrasound and targeted drug delivery, with applications in the GI tract.

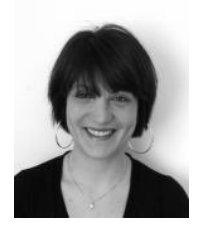

Antonella Verbeni joined the Scuola Superiore Sant'Anna, Pisa, Italy, working on surgical robotics focusing her activity on endoscopic capsules. She received her Master Degree in Biomedical Engineering at the University of Pisa, Italy, in 2011. She is currently a PhD student in Biorobotics at The BioRobotics Institute of the Scuola Superiore Sant'Anna in Pisa, Italy.

Her main research interests include the study and the applicability of therapeutic ultrasound.

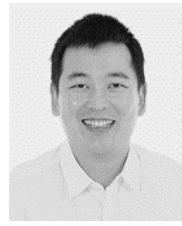

Yongqiang Qiu received the B.Eng. degree in biomedical engineering from Tianjin University, China, in 2007, and the MSc degree in biomedical engineering and Ph.D. degree in mechanical engineering from University of Dundee, U.K., in 2009 and 2014, respectively. After his Ph.D., he worked in the Institute for Medical Science and Technology, School of Medicine, University of Dundee, U.K., until moving to the School of Engineering, University of Glasgow, U.K., in late 2015. His research interests include the development of high frequency ultrasonic devices for biomedical and life sciences applications, such as acoustic particle/cell manipulation and microultrasound imaging. He is a member of IEEE since 2010.

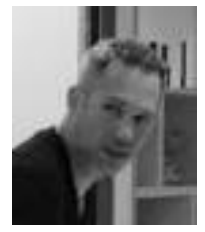

Ben F. Cox recived his BSc from Dalhousie University in Canada. He became involved in medical research at Massachusetts General Hospital in the USA. He then went on to complete his medical studies at the First Faculty of Medicine, Charles University in the Czech Republic.

After graduating from medical Ben became involved in interventional imaging research specifically in the area of MRI. $\mathrm{He}$ is currently the Clinical Research Fellow on the Sonopill programme.

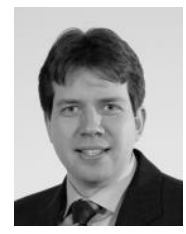

Jan B. Vorstius is a lecturer in the discipline of Mechanical Engineering. He obtained his undergraduate degree in Biomedical Engineering at the University of Applied Sciences in Gelsenkirchen, Germany in 2008. He then came to the University of Dundee where he obtained his $\mathrm{PhD}$ for his research on a modular bioreactor design for muscle tissue engineering in 2012.

In 2010 Dr Vorstius was appointed as a Lecturer and teaches within the undergraduate Mechanical Engineering and the postgraduate Biomedical Engineering programs, especially Control Engineering, Thermodynamics, Circuit Design and Biomedical Systems. Since 2015 he is the programme director for the mechanical Engineering programmes at the University Dundee. 


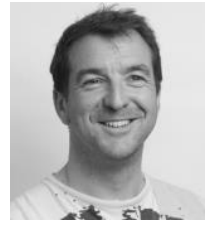

Ian P. Newton received his BSc. degree from the University of Stirling, in 1991. From 1992, he was a researcher at the University of Dundee, and has worked on various projects. As Senior Scientific Officer in the Nathke laboratory he now has over 23 publications.

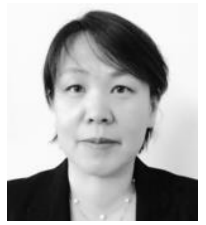

Zhihong Huang graduated with a B.Sc. degree in instrumentation from Tianjin University, China, and a Ph.D. degree in mechanical engineering from the University of Glasgow. She is currently Professor of Biomedical Engineering and Associate Dean for Internationalisation in the School of Science and Engineering, University of Dundee. Building upon experiences in vibration analysis, dynamic modeling and simulation, and optics, she is particularly interested in cross-disciplinary research in the field of biomedical engineering. Her main research emphasis is on activities in the development of high-power ultrasonic technology, high-intensity ultrasound, and optical coherence tomography with elastography.

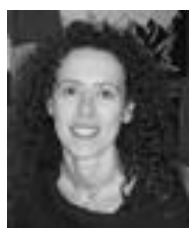

Arianna Menciassi received an M.Sc. degree in physics (Hons.) from the University of Pisa, Italy, in 1995, and the Ph.D. degree from the Scuola Superiore Sant'Anna (SSSA), Pisa, in 1999. She is a Full Professor of biomedical robotics and bioengineering at the SSSA. Her main research interests include medical mechatronics, biohybrid systems, biomedical micro and nanodevices, and robotic surgery. She possesses extensive experience through European projects and international collaborative projects on topics related to robotic and microrobotic diagnosis, surgery and therapy. She is the author of about 370 international papers (Source: Scopus), with more than 200 ISI journals, one edited book, and six book chapters on medical devices and microtechnologies. She is also inventor of 25 patents, national and international.

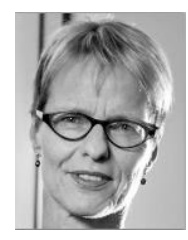

Inke Näthke received her Ph.D. from the University of California San Francisco in 1991. She completed postdoctoral studies at Stanford and Harvard University Medical Schools. At Stanford she worked on cadherin-mediated cell adhesion and the role of the adenomatous polyposis coli protein (APC) in epithelia. At Harvard she focused on the molecular interactions between APC and the microtubule cytoskeleton. Since 1998 she has been a Principle Investigator at the School of Life Sciences at the University of Dundee where she was awarded a personal chair in 2007. She is currently Professor of Epithelial Biology, Deputy Head of the Division of Cell and Developmental Biology, and Associate Dean for Professional Culture.

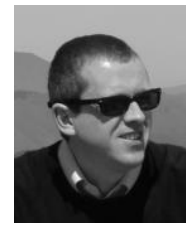

Sandy Cochran received his B.Sc., Ph.D. and M.B.A. degrees from the University of Strathclyde, in 1986, 1990 and 2001 respectively. Following work in various research and academic roles from $1990-2005$ at the University of Strathclyde and University of the West of Scotland (UWS), he became full Professor at UWS in 2005, subsequently moving to the University of Dundee and becoming a Professor there in 2010. He is now Professor of Ultrasound Materials and Systems in the School of Engineering, University of Glasgow. He is the author of over 300 technical publications, proceedings, editorials and books. His research interests include new piezoelectric materials for ultrasonic transducers, the transducer devices themselves and their applications in medicine and industry. He is Vice-chair of the Technical Program Committee of the IEEE International Ultrasonics Symposium, leading Group 5, Transducers and Transducer Materials and an active member of the UK Institute of Physics. 